\title{
Editorial Column
}

\section{Global Health Services Research: Challenging the Future}

Today health and global well-being are inextricably intertwined. Yet much of the data and research necessary to fashion good health policy is either inadequate or missing altogether. This special edition of Health Services Research testifies to the emerging recognition of the importance of global health services research and constitutes a timely addition to the body of research needed to help guide good policy.

Last year, as SARS raced around the world, it was painfully clear that health is a central global concern. The rapid transnational spread of new and emerging infectious diseases and their potential use as terrorist agents grab headlines, while in many countries-indeed in whole regions of the world such as sub-Saharan Africa-economic, political, and social development is retarded because of poor health and poor health care. This threatens civil societies, and even national and regional stability. ${ }^{1}$ It also challenges the organization and financing of both government and private sector health care systems. At a personal level the consequences of disease and poor health are felt every day.

The risks of failing to address the problems of disease, poverty, and unrest, paired with the opportunities for constructive health policy change, prompts a powerful cry for proactive efforts and for greater resources to conduct the research needed to guide that policy.

\section{The Challenge facing Health Services Research}

The challenge is daunting. Original concerns over the impacts of infectious diseases like cholera in the 1800 s led to international cooperation and to study on infectious diseases and their impacts on poor countries. But the challenges are not limited to acute disease. The prevalence of chronic disease, both in developing and more developed countries, is growing dramatically, placing additional burdens on already stretched delivery systems. The economic consequences of poor health in many areas have also been documented, but the debate continues about the causal relationship between health and economic developments. ${ }^{2}$ Recent releases such as the WHO's Millennium 
Development Goals ${ }^{3}$ and the Macro Economics Report on Heath have added heightened urgency with respect to the need and potential for action. ${ }^{4}$

Yet in a sense, issues relating to healthcare services are only the tip of the iceberg. Trade and tax policy, sometimes even more than health care, can and do have major impacts on the health of people as well as on the financing available to fund programs. Often the effects of economic activity have unintended health consequences-both negative and positive. For instance, in Africa and elsewhere, the building of roads to support commerce and product delivery had the unintended consequence of facilitating the spread HIV/AIDS as truck drivers used the services of commercial sex workers along the transportation networks. Tobacco represents one particularly important and well-studied health issue relating to development, but there are many others.

In recent years, the relationship between health and foreign policy has also come to the attention of health researchers. Health care represents an area of shared human values among (local/indigenous) people and health professionals where constructive engagement neutral to religion, culture, and politics is possible. Emphasizing health would tend to refocus foreign policy on humanitarian objectives instead of on the more traditional concerns of defense and economics. Yet NGOs in Iraq and elsewhere have been reluctant to have outside national governments involved because they fear it will make their jobs more difficult and less safe if healthcare-once only associated with altruism-becomes part of foreign policy.

As Amartya Sen has so eloquently pointed out, health is closely linked to social, political, and personal freedom. ${ }^{5}$ Health is increasingly seen as an essential function of government, and the consequences of unchecked diseases such as HIV, TB, and malaria impede the development of civil societies, sap public budgets, often lead to unrest, and can even threaten national and regional stability.

Health services researchers are also paying increasing attention to health as a global public good, the links between health and migration, the education of women, political leadership, quality of care, the environment, and social justice.

This growing list of health-related research interests does not lessen the policy importance of long-studied areas such as infectious diseases, health and development, financing, reproductive health, access to care, and public health. Nor does increased interest mask the fact that the basic data necessary to inform good policymaking are often unavailable. Equally unavailable is the funding required to carryout this desperately needed research. 
Our challenge as health services researchers is to develop new data sets and methods, to rigorously conduct required studies, and to become advocates for increased health research funding. We must underscore the high return that such research dollars can yield both in greater efficiency in use of limited public funds and in increased well-being of people. We should also advocate for processes such as the use of the mandatory health impact assessment required in the European Union (EU) for health-related projects. This requirement is now written into the agreements that govern operation of the EU and requires that major policy changes including those that are not specifically health related (e.g., a trade policy) be assessed for their impact on the health of the public. ${ }^{6}$

\section{THIS VOLUME}

We believe this volume is a timely addition to the increasingly global nature of health care research. It recognizes that we have a tremendous amount to learn from each other and from the experiences of other countries and communities. It is also a testament to the increasing volume of high quality health services research being conducted in many disparate institutions around the globe. We received more than 50 submissions for this volume-ample evidence of interest and activity worldwide.

Although we have selected the best research submitted to us regardless of area, we are pleased that the articles included this volume represent tremendous diversity both geographically and in areas researched. Geographic areas range from Taiwan and Thailand, through India in Asia to Uganda in Africa, to the former Soviet Union, and Macedonia. A number of the studies deal with multiple countries in Africa and the OECD. Issue areas of research also vary, and methods include both quantitative and qualitative approaches.

This volume focuses on pressing research needs in four areas. The section on cross-country studies addresses important international issues such as utilization, quality of care, and long term care expenditures. This section also constitutes recognition that developed countries such as the United States and those in the EU have much to learn from each other and from the experiences and experiments in other parts of the world. The paramount importance of public health is recognized in articles on HIV, public versus private use of services in India, and the prevention of genocide. Important contributions to reproductive health, which is essential in developing countries, are examined 
in the third section. The final section focuses on the critical issues of financing and cost.

Studies included also address some new and nontraditional areas that are increasingly concerns of health policy research, for example, the intersection of health and the prevention of genocide, informal caregivers, and the use of local midwives.

We hope this volume aids policymaking, spurs further investigations, and heightens the commitment of the health services research community and funders to international health care research. We trust it furthers the growing recognition that health and health care are areas around which diverse people and communities can constructively engage, despite cultural divides, to address issues that we all share as global citizens.

\section{Ross Anthony \\ Nicole Lurie \\ Guest Editors}

\section{ACKNOWLEDGMENTS}

The editors would like to thank the Martin Foundation, Carol and David Richards, and Kaiser Permanente for their support which helped fund this effort.

\section{NOTES}

1. Peter Chalk and Jennifer Brower, The Global Threat of New and Reemerging Infectious Diseases: Reconciling U.S. National Security and Public Health Policy, RAND, MR-1602-RC, 2003.

2. David Bloom and David Canning, "The Health and Wealth of Nations," Science, Volume 287, February 18, 2000.

3. World Bank, "The Millennium Development Goals," www.developmentgoals.org.

4. WHO, Report of the Commission on the Macroeconomics and Health: Investing in Health for Economic Development, WHO, 2004.

5. Amartya Sen, Development as Freedom, Random House, New York, 1999.

6. These assessments are designed to inform the public about the health consequences of policy changes but do not have the force of law as is the case for rulings such as those issued by the US Environmental Protection Agency. 